\title{
Characterization of the volume and thickness of DIEP flap by CTA image processing
}

\author{
$1^{\text {st }}$ María Alejandra Cerón \\ Dept. of Mechanical Engineering \\ Universitat Politécnica de Catalunya \\ Barcelona, Spain \\ maria.alejandra.ceron@upc.edu
}

\author{
$4^{\text {th }}$ Ana López Ojeda \\ Plastic Surgery Department \\ Hospital Universitari de Bellvitge \\ Barcelona, Spain \\ ablopez@bellvitgehospital.cat \\ $7^{\text {th }}$ Hernán A. González Rojas \\ Dept. of Mechanical Engineering \\ Universitat Politécnica de Catalunya \\ Barcelona, Spain \\ hernan.gonzalez@upc.edu
}

\author{
$2^{\text {nd }}$ Julián Loaiza Duque \\ Dept. of Mechanical Engineering \\ Universitat Politécnica de Catalunya \\ Barcelona, Spain \\ julian.david.loaiza@upc.edu
}

\author{
$5^{\text {th }}$ Marta Alcover Morro \\ Plastic Surgery Department \\ Hospital Universitari de Bellvitge \\ Barcelona, Spain \\ marta.alcover@bellvitgehospital.cat \\ $8^{\text {th }}$ Antonio J. Sánchez Egea \\ Dept. of Mechanical Engineering \\ Universitat Politécnica de Catalunya \\ Barcelona, Spain \\ antonio.egea@upc.edu
}

\author{
$3^{\text {rd }}$ Sergi Barrantes Verdoy \\ Plastic Surgery Department \\ Hospital Universitari de Bellvitge \\ Barcelona, Spain \\ sergi.barrantes@bellvitgehospital.cat \\ $6^{\text {th }}$ Pedro Quetglas Barea
Plastic Surgery Department
Hospital Universitari de Bellvitge
Barcelona, Spain
pquetglas@ @ellvitgehospital.cat
}

\begin{abstract}
Breast cancer is the most commonly diagnosed cancer worldwide and is the leading cause of cancer-related death in more than 100 countries. Breast cancer surgery, especially when it involves a mastectomy, is associated with unaesthetic results that can be traumatic. Therefore, breast reconstruction is crucial for the patient to return to normal life, avoiding the psychological consequences. Based on free tissue transfer with microsurgery, autologous breast reconstruction is the gold standard for breast reconstruction, especially in irradiated patients. To plan the reconstruction surgery and locate the cutaneous perforating vessels supplying blood to the flap, preoperative Computed Tomography Angiography (CTA) is usually performed. However, only approximate and qualitative measurements are obtained and the location of the umbilical perforators reported by the radiologist. This paper advances a quantitative method to assess autologous Deep Inferior Epigastric Perforator (DIEP) flap volume and thickness from CTA images. This method is validated by measuring flap volume intraoperatively in the operating room of the Hospital Universitari de Bellvitge. These measurements could improve preoperative planning by reconstructive surgeons as they would know beforehand whether the amount of adipose tissue that can be harvested is sufficient to reconstruct the breast completely. This information could be crucial in thin and largebreasted women or if bilateral breast reconstruction is planned.
\end{abstract}

Index Terms-CTA, DIEP flap, image processing, volume, thickness.

\section{INTRODUCTION}

Breast cancer is characterized by the uncontrolled growth of malignant cells in the mammary epithelial tissue [1]. Treatment comprises different management strategies such as chemotherapy, radiotherapy, hormonal therapy, and im- munotherapy [2]. Despite recent advances in other therapy modalities, surgery is still the most effective treatment for nonmetastatic breast cancer. This kind of surgery includes both tumor excision and axillary lymph node management. Regarding the breast, surgical options can be categorized by Lumpectomy (breast-conserving surgery that only removes the part of the breast that contains malignant tumor along with some healthy tissues, generally in the initial phases of cancer) and Mastectomy, which includes excision of the whole mammary gland, with the removal or sparing of the nipple, surrounding skin, or even the pectoralis major, depending on the case. Mastectomy has dramatic psychological effects on women who undergo the procedure. The loss of a breast leads to a feeling of asexuality and loss of self-image, and consequent depression in most women [2]. Whenever it is possible, and for those who desire it, breast reconstruction is offered. It can be either immediate (at the time of mastectomy) or delayed (at a later time) and, it aims to restore breast contour, as it has shown to have a profound positive impact on the mental health of these patients, and, subsequently, a better quality of life [3], [4].

Regarding breast reconstruction techniques, they can be divided by the use of implants or autologous tissue reconstruction. In general terms, tissue expanders and implants provide a faster, less morbid procedure for the patient. However, postoperative complication rates are high and aesthetic results are poor compared with other techniques [3]. Moreover, radiotherapy has been shown to play a crucial part in implant failure due to exposure, infection, and contracture, as the chronic 
damage produced to the thoracic wall and surrounding tissues critically alters its physiologic and healing capacities [5]. For these reasons, implant breast reconstruction is far from ideal in patients who will receive radiotherapy. On the other hand, autologous breast reconstruction is based on flaps, which are transfers of tissue from the patient (these come from the lower abdomen, the back, buttocks, or inner thighs) that can be applied to supply a function that has been lost, in this case, skin cover and breast contour. The tissue can be separated from its original blood vessels and transferred to its new place in the chest. It is frequently called free flap [6], or the tissue can remain attached to its original blood vessels and moved under the skin to the chest that, in this case, it is called a pedicled flap. There is evidence that autologous reconstruction offers a more aesthetically pleasing and better functional outcome in the long term [7], [8], and it is the technique of choice whenever radiotherapy is scheduled [9].

Nowadays, there are multiple flaps available for breast reconstruction. However, the Deep Inferior Epigastric Perforator (DIEP) flap has become the option of choice in most cases thanks to the large availability of fatty tissue in the lower abdomen, consistent and long vascular pedicle, and improved contour waist after the flap has been raised from the abdomen as the donor site is closed in a manner that is similar to an aesthetic abdominoplasty procedure. This free flap consists of the skin and fatty tissue from the lower abdomen that is transferred to the breast and reshaped. Its blood supply arises from the Deep Inferior Epigastric Artery (DIEA). The DIEA is a direct branch from the external iliac artery that moves along the posterior aspect of the rectus abdominis (RA) muscles [10]. Once harvested, DIEA and vein are connected to the internal mammary vessels using microsurgical techniques, and blood supply is restored. The DIEA is responsible for vascularizing the skin and fat of the lower abdomen through small vascular branches that pierce the RA muscles and sheath and irrigate the area. These branches are known as cutaneous perforators from the DIEA. They are more likely to be found surrounding the umbilicus, and the whole lower abdomen can be irrigated through only one perforator if it is raised as a flap. Even though the DIEP flap surgery has become more popular for breast reconstruction, it requires significant microsurgical experience to harvest because involving a meticulous dissection of the vessels within the rectus abdominis muscle [11]. According to that, during surgical planning, some considerations should be taken into account. These are such as the localization of perforators, the Superficial Inferior Epigastric Vein (SIEV) localization, the pedicle length, the intramuscular course of the pedicle, the artery caliber, the artery diameter at the sheath and before joining the Iliac artery, and the fatty tissue volume available to be harvested. Based on these conditions, the surgeon will plan the surgery accordingly and decide the best perforator for irrigating the soft tissue used for breast reconstruction. The whole point of the DIEP flap [12] is to preserve abdominal muscles and have certain advantages related to lower rates of donor site complications and functional impairment, reduction of in-hospital stay, and a better cosmetic appearance [13].
For these reasons, the DIEP flap is considered to be the gold standard in breast reconstruction.

Three-dimensional reconstruction images of the deep inferior epigastric perforators are obtained by Computed Tomography Angiography (CTA). The CTA is a standard method of preoperative assessment of abdominal vascular anatomy, which has proved to reduce the time in the operating room table [14], and reduces the risk of complications, and improves the efficiency of flap harvest [15]. Particularly, CTA reduces operating time by a mean of 58 minutes (95\% Confidence interval (CI) 25 to 91 minutes) when a perforator mapping is performed, appearing superior to another imaging modality such as the Ultrasound. Also, a novel aesthetic surgery simulator software has been developed to aid the surgeons in determining the geometry of the flap, customize threedimensional printed breast molds, and reduce surgical time and issues of autologous breast reconstruction [16]. Besides, the patient can have an aesthetic overview of what the breast will look like after reconstruction. Also, Zhang et al. [17] reported an analysis of CTA data of patients who received postoperative treatment for breast cancer to determine the need to use superficial epigastric artery (SIEA) flaps. They concluded that the SIEA is recommended when one of the two criteria are matched, patients with a Body Mass Index (BMI) $\geq 25 \mathrm{~kg} / \mathrm{m}^{2}$ or an SIEA diameter $\geq 2.0 \mathrm{~mm}$. Also, Rosson et al. [18] used CTA to estimate the preoperative volume and weight of the DIEP flap. These estimated parameters were compared with the actual intraoperative volume and weight for each patient to determine if a potential flap to be harvested can be accurately identified using CTA. They measured the height and width of the flap with a ruler, mapped it on the patient, and used fiducial markers placed on the flap mapped before the CTA scanning. After that, the software was used to draw the volume of interest (based on the mentioned markers) and set the evaluation limits. Later the program calculated the volume of the area of interest. It was considered as the estimated volume and then was assumed to be the estimated weight. They showed a $99.7 \%$ accuracy results in the average estimated weight of the flap concerning the actual weight. Even though they accurately narrowed down the adipose tissue region of the flap with fiducial markers, they drew the flap limits manually in the software, and there is no information about how the estimated parameters were calculated. This work-based its procedure on the flap mapped on the patient to see how accurately the surgeons assessed the viable flap.

Consequently, following the last research topic, this work focuses on reconstructing the Deep Inferior Epigastric Perforator flap using CTA images processing algorithms. Therefore, the aim has been to develop a quantitative method to address two relevant parameters: the volume and thickness of the DIEP flap. Additionally, the measurement of the flap volume will be validated in the operating room to determine the accuracy of the estimation. Therefore, the method is intended to help surgeons decide the optimal procedure for autologous tissue surgery by estimating the DIEP flap volume and thickness. Furthermore, to determine if the patient can be considered a 
candidate for the autologous procedure and decide the best procedure according to her anatomy.

\section{Methodology}

\section{A. Patients and dataset description.}

The dataset used in this study includes about five patients with a CTA of the abdominal section who underwent DIEP flap breast reconstruction at the Hospital Universitari de Bellvitge in Barcelona. Table I shows the demographic data, type of procedure (mastectomy side), and projected DIEP flap area to be cut, defined by the width and the height of an elliptical trajectory, before it is harvested from the abdomen of each patient. The images used in this project were acquired through two Computed Tomography scanners in which the patient was in the supine decubitus position. The first scanner, an Aquilion ONE CT (Canon), includes anatomical sequences of abdominal CTA in an axial plane in which between 410 and 556 slices were obtained using a slice thickness of $1 \mathrm{~mm}$ and the following acquisition parameters: $100 \mathrm{KVp}, 120 \mathrm{~mA}$ of the X-ray tube current, $500 \mathrm{~ms}$ of the exposure time, and $600 \mathrm{mAs}$ of the exposure. The second scanner corresponds to a LightSpeed VCT CT scanner (GE Medical Systems). Abdominal CTA anatomical sequences were performed in an axial plane obtaining between 345 and 730 slices using a slice thickness of $1,25 \mathrm{~mm}$, a spacing between slices of $0,625 \mathrm{~mm}$, and the following acquisition parameters: $100 \mathrm{KVp}, 150 \mathrm{~mA}$ of the X-ray tube current, $600 \mathrm{~ms}$ of the exposure time, and $2 \mathrm{mAs}$ of the exposure. Iomeprol was used as a contrast with a total volume and flow rate of $100-120 \mathrm{ml}(400 \mathrm{mg} / \mathrm{ml})$ and $4 \mathrm{ml} / \mathrm{s}$, respectively.

\section{B. Preprocessing and image filtering}

Preprocessing CTA images starts with a low-pass filter to smooth and remove unwanted image features. An adaptive median filter is implemented for this purpose, which performs spatial processing to preserve details and smooth non-impulsive noise and not eroding edges or other small structures [19]. The filter compares each pixel with the surrounding pixels and replaces the pixel that differs from the majority of the neighboring pixels with the median values of the surrounding pixels. This process is repeated until all noisy pixels are removed. As a result, a smoothed image is obtained. Subsequently, an unsharp masking technique is performed to make an image sharper by subtracting a blurred version of the image. Once the enhanced image is obtained, a thresholding technique is implemented using the method of

TABLE I: Demographic data and the projected area to cut of the DIEP for the treated patients.

\begin{tabular}{|c|c|c|c|c|c|c|}
\hline Patient & 1 & 2 & 3 & 4 & 5 & Mean \pm STD \\
\hline Age [years] & 56 & 52 & 43 & 43 & 54 & $49.6 \pm 6.2$ \\
\hline BMI $\left[\mathrm{kg} / \mathrm{m}^{2}\right]$ & 31.25 & 29.97 & 33.46 & 31.63 & 19 & $29.1 \pm 5.8$ \\
\hline Breast side & Left & Left & Left & Left & Left & - \\
\hline Prev. surgery & No & No & No & $\mathrm{Yes}^{a}$ & Yes $^{a}$ & - \\
\hline Planned flap $^{b}$ & $38 \times 15$ & $39 \times 14.5$ & $41 \times 15$ & $40 \times 15$ & $28.5 \times 10$ & - \\
\hline
\end{tabular}

${ }^{a}$ Previous mastectomy to this autologous surgery. ${ }^{b}$ The planned DIEP flap is defined by the total width $[\mathrm{cm}]$ and length $[\mathrm{cm}]$ of the drawn flap at the patient's umbilicus.
Otsu [20] to extract the torso and other features of interest. This method is based on a non-parametric procedure using histogram statistics for optimal thresholding by minimizing the variance between classes through an exhaustive search with which it is possible to obtain a binarized image. The algorithm assumes two basic classes of pixels: foreground and background pixels. The algorithm then disconnects classes by enumerating the optimal threshold so that their combined variance (intra-class variance) is minimal. The method of Otsu can be extended by assuming different thresholds that divide the image into different classes, called multilevel thresholding [21]. Finally, the binarized images are combined with morphological operators to separate the patient's torso from its surrounding environment so that it is subsequently possible to delineate and extract the flap (see Fig. 1).

\section{Characterization of the DIEP flap}

In the DIEP flap surgery, the width and height of the flap are defined using the umbilicus as a central reference point (see Figure 2). The upper horizontal line is drawn just above the umbilicus up to the level of the Iliac crests or surpassing them in some patients. This determines the total width of the flap with typical values between 25 and $40 \mathrm{~cm}$. The length of the flap is traced at 10 to $15 \mathrm{~cm}$ from the upper edge of the umbilicus [22]. Subsequently, the proximal points of each line are connected as a continuous curved line. In the algorithm, the width and length are required as input parameters. Firstly, the algorithm works on identifying and selecting slices that show the umbilicus in a coronal plane. Then, it determines the umbilicus spatial coordinates and identifies the umbilicus location in the axial plane to discard unnecessary slices above it. Likewise, in the axial slice located at the upper limit of the umbilicus, the method of Canny [23] is used to detect the edge of the torso. The pixels that sum up to the desired width input value of the flap are preserved. For this process, the algorithm divides by two the desired width value and, based on the centroid, the pixels (in centimeters) in both directions (left and right) are summed to complete the width input value discarding the remaining pixels of the detected edge. Then, the algorithm delimits the width of the flap by setting marks, which are adjusted in a coronal plane at the height of the Iliac crests approximately. On the other hand, a similar process is performed for the flap length, calculating the length from the lower part on the umbilicus to $11-14 \mathrm{~cm}$ downwards in a coronal plane. Finally, on this plane, the proximal points

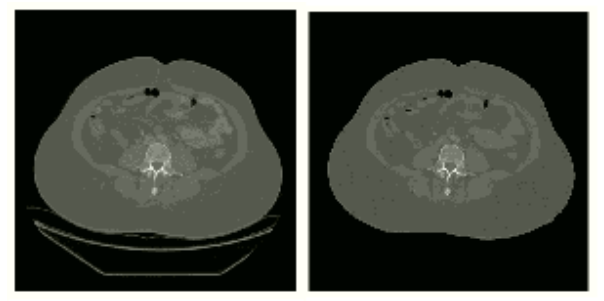

Fig. 1: (Right) The original CTA image. (Left) The filtered image and extracted torso. 


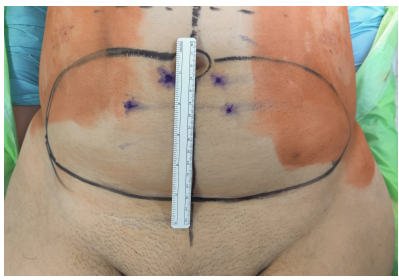

Fig. 2: The drawn area of the planned DIEP flap to be cut.

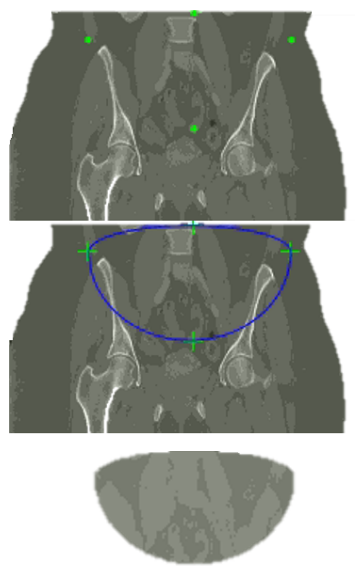

Fig. 3: (Up) The point sites of the DIEP flap in the coronal plane. (Middle) The points are connected as a curved line. (Down) The extracted DIEP flap by image processing.

are connected to be delineated as an ellipse (see Fig. 3) that corresponds to the drawn flap area on the abdomen of the patient. Equation (1) of the elliptical trajectory is defined as follow:

$$
\frac{x^{2}}{a^{2}}+\frac{y^{2}}{b^{2}}=1 ; \quad b=\left\{\begin{array}{cc}
b_{\text {sup. }}, & y \geq 0 \\
b_{\text {inf. }}, & y \leq 0
\end{array}\right.
$$

\section{Volume of the DIEP flap}

The volume of the harvested DIEP flap was measured in the operating room just before being transplanted to the breast using a graduated bucket with a capacity of $5000 \mathrm{~cm}^{3}$ and $\pm 100 \mathrm{~cm}^{3}$ of precision. The bucket was previously sterilized with plasma and filled with $2000 \mathrm{~cm}^{3}$ of physiological saline solution. Figure 4 shows the graduated bucket and the procedure to measure the volume of the DIEP flap. Regarding the algorithm, the drawn area of the flap is extracted from the rest of the body at the coronal CTA image to works only with the corresponding axial slices in the area of interest. This is performed by a region of interest (ROI) that is converted to a binary mask and subsequently as an uint 16 image. This mask is multiplied with the filtered images in the coronal plane, and only the voxels that correspond to 1 in the mask are preserved, and the voxels with 0 are eliminated. Next, the pixels in this area are summed and converted to calculate the total volume of the flap in $\mathrm{cm}^{3}$.

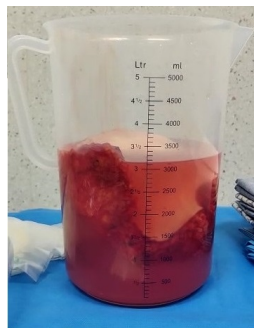

Fig. 4: The measurement of a DIEP flap with a graduated bucket filled with physiological saline solution.

\section{E. Thickness of the DIEP flap}

The axial images correspond to the area of interest are segmented by masking all elements below the surface of the RA sheath to identify the adipose tissue. Then, the algorithm locates three points in the coronal plane where the segmented umbilicus is. These points are located at a distance of $5 \mathrm{~cm}$ away from the umbilicus centroid in the following directions: left, right, and down. Later, the algorithm takes axial slices (equivalents to the $\mathrm{Y}$ coordinate of the three points in the coronal plane) to measure the thickness following the same procedure reported by Woo et al. [22]. Thus, the thickness is calculated from the skin outline to the RA sheath and is an average value due to the thickness measurements at the three points.

\section{RESULTS AND DISCUSSION}

The preliminary results of this work can be appreciated by comparing the volume obtained by the image processing algorithm with the results measured during surgery. Table II shows the measuring volume and the volume estimated by the algorithm, as well as the deviation errors between the values. Table III shows the thickness values of the flap at different sites on the abdominal wall and the mean for each patient.

In Table II, there are two types of measured volume values: the recorded and estimated. These are calculated in the operating room and by the algorithm, respectively. According to the results, the measured volumes are similar in the different patients except in one case, in which the measured volume is below $1000 \mathrm{~cm}^{3}$. The above is due to the BMI of the patient (see Table I), which is below the rest of the subjects,

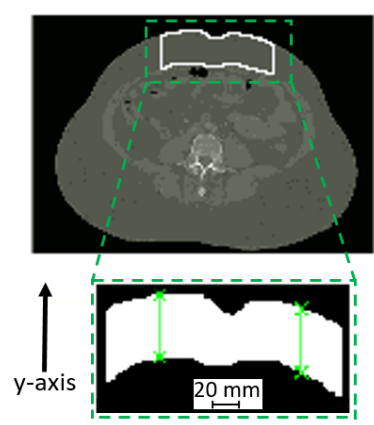

Fig. 5: The measurement of the DIEP flap thickness at the adipose tissue region from an axial view. 
TABLE II: The recorded and estimated DIEP flap volume from patients.

\begin{tabular}{|c|c|c|c|}
\hline & \multicolumn{3}{|c|}{ DIEP flap volume } \\
\hline Patient & $\mathbf{R}^{a}\left[\mathbf{c m}^{3}\right]$ & $\mathbf{E}^{b}\left[\mathbf{c m}^{3}\right]$ & Deviation error [\%] \\
\hline 1 & 1500 & 1513 & +0.86 \\
\hline 2 & 1300 & 1225 & -5.76 \\
\hline 3 & 1900 & 1863 & -1.95 \\
\hline 4 & 1500 & 1625 & +8.33 \\
\hline 5 & 300 & 284 & -5.34 \\
\hline
\end{tabular}

${ }^{a}$ Recorded. ${ }^{b}$ Estimated.

who are considered obese type 1 . The estimated volume is denoted a significant variability in the deviation error between the patients, which is mainly attributed to the different cutting trajectories used by surgeons for harvesting the DIEP flap. The worst-case scenario is a deviation error of $+8.33 \%$ of the volume estimation concerning the recorded one, whereas the best approach is found to be $+0.86 \%$. In Table III, the thickness was different for each patient because of the quantity of adipose tissue in the lower abdomen. The measurements of the right and left sides are not closely distinct from the low point. As expected, patient 5 has a smaller flap thickness than the rest of the subjects due to his significantly lower BMI.

The main difference between the measured and estimated DIEP flap volume is due to the cutting trajectory of the flap, which is why some researchers have proposed different tools to evaluate the flap size [25]. The exact dimensions and shape of the excision are crucial parameters to assess to improve the method accuracy, although, in this first approach, we have used a single elliptical trajectory to determine the DIEP volume. We intend to determine the variability of this procedure when different curvature trajectories are used. Currently, the procedure for harvesting the flap is made based on the experience of the surgeon [26]. The incision lines are drawn based on various factors, including height, waist perimeter, skin laxity, amount of fatty tissue needed, and the desired position of the future scar, which is intended to be placed hidden by the underwear. Nevertheless, the elliptical shape of the incision is similar in all cases and can be estimated by its height and width.

The amount of skin and fat volume harvested has important implications. Generally, the amount of tissue in the ellipse of a DIEP flap is greater than the volume needed to reconstruct a single breast. In fact, in most cases, half of the volume would be enough. Before insetting the flap in the chest, the surgeon excises the lateral borders of the tissue, which are less irrigated than the tissue around the cutaneous perforator, to minimize the risk of fat necrosis. The purpose of the ellipse

TABLE III: The thickness values of the DIEP flap for each patient at the three region of interest.

\begin{tabular}{|c|c|c|c|c|}
\hline & \multicolumn{4}{|c|}{ Thickness DIEP flap } \\
\hline Patient & $\mathbf{R}^{a}[\mathbf{m m}]$ & $\mathbf{L}^{b}[\mathbf{m m}]$ & $\mathbf{I}^{c}[\mathbf{m m}]$ & Mean \pm STD [mm] \\
\hline 1 & 45.8 & 45.8 & 36.1 & $42.6 \pm 5.6$ \\
\hline 2 & 33.0 & 32.1 & 39.4 & $34,8 \pm 3.9$ \\
\hline 3 & 45.9 & 48.5 & 44.1 & $46.2 \pm 2.2$ \\
\hline 4 & 50.7 & 49.7 & 54.6 & $51.7 \pm 2.6$ \\
\hline 5 & 15.6 & 16.3 & 13.5 & $15.1 \pm 1.45$ \\
\hline
\end{tabular}

${ }^{a}$ Right point. ${ }^{b}$ Left point. ${ }^{c}$ Inferior point. shape is to allow for appealing wound closure that otherwise would be impossible or aesthetically unpleasing. Nevertheless, in patients suffering from genetic mutations leading to hereditary breast cancer, the most common being BRCA1 and BRCA2 [27], often bilateral mastectomy is necessary to reduce the risk of breast cancer in the contralateral gland. If bilateral mastectomy is planned, a bilateral DIEP can be harvested [28]. The skin incision is similar to the classic DIEP, but two cutaneous perforators are selected and dissected to the Deep Inferior Epigastric Artery (DIEA), one for each side of the flap. The flap is then split vertically at the middle line, and two separate flaps are obtained, one for each breast. Doubts may arise whether the amount of fat in the lower abdomen is sufficient to perform a bilateral reconstruction, especially for skinny women with large breasts. In such cases, the tool proposed in this work could be paramount to determine whether this type of reconstruction is advisable, as it would indicate the maximum amount of fat volume and the maximum thickness of the flap that can be harvested. If the volume is insufficient, another type of reconstruction should be taken into consideration. On the contrary, if the volume harvested is excessive, especially in patients with low BMI and nondistensible skin, wound closure can lead to skin tension, which can result in a high scar, which can be unpleasing because it cannot be hidden under the underwear, or even wound dehiscence and infection, which are considered major complications of the procedure [29]. The tool proposed by this article could help surgeons to estimate the flap dimensions that assure sufficient volume while minimizing the risk of wound tension. Finally, the automatization of DIEP volume and thickness could serve research purposes. Factors such as body weight and high BMI are associated with significant complications in DIEP reconstruction [30], such as abdominal wall weakness, fat necrosis due to insufficient blood supply to the flap, venous thrombosis, systemic complications, and longer hospitalization stays. Automated retrospective analysis of volume and thickness of the patients could help to identify those in greater risk for complications.

We will pretend to replicate the algorithm with other patients in future works as soon as more CTA is recorded and measurements in the operating room are available. Besides, we pretend to analyze the correlation of the flap volume with other parameters such as the pedicle length, the deep inferior epigastric artery caliber and length, and the length and caliber of perforators because these parameters will be relevant to make decisions during DIEP surgery planning. It is essential to validate and improve the algorithm performance through feedback from using it on other patients. Also, we will analyze the possibility of using some algorithms based on organ and Atlas segmentation [31], [32] that can support us to segment accurately the arteries and perforators by reducing the variability in the delineation of the body part. Likewise, we plan to complement the algorithm with a $3 \mathrm{D}$ reconstruction of the abdomen surface of the patient. With this reconstruction, plastic surgeons could choose the height, width, and degree of curvature of the planned ellipse and be aware of the amount of 
adipose tissue available to harvest, changing these parameters whether the planned volume is excessive or insufficient.

\section{CONCLUSION}

We have demonstrated the feasibility of an accurate measurement of the DIEP flap by using a CTA image processing algorithm. The image processing protocol is implemented in Matlab, giving immediate results for the clinician from the width and length as input parameters. This is to automatically set the initial abdominal markings for preoperative planning since the volume and adipose tissue thickness for breast reconstruction will be assessed beforehand. The proposed method predicts the volume DIEP flap with an accuracy of $91.67 \%$ in the worst-case scenario. It also indicates the thickness of the flap in different regions of interest. Additionally, in future works, we intend to develop a sufficiently robust image processing algorithm to determine the length and caliber of the epigastric tube used in autologous breast reconstruction. Automated DIEP flap characterization using CTA data could help surgeons improve aesthetic results and minimize complications, save time and resources to the radiologists, and serve for research purposes for retrospective studies.

Compliance with Ethical Standards: All the patients were informed of the procedure and asked to sign a consent form to use their data for research purposes. The Ethics Committee of Hospital Universitari de Bellvitge approved this study under the ethical standards laid down in the Declaration of Helsinki and its later amendments.

\section{ACKNOWLEDGMENT}

This work is supported by the Serra Húnter program (Generalitat de Catalunya) reference number [UPC-LE-304 (2018)] and by the Plastic Surgery Department of the Hospital Universitari de Bellvitge.

\section{REFERENCES}

[1] K.E. Lukong, "Understanding breast cancer - The long and winding road”. BBA Clinical, vol. 7, pp. 64-77, 2017

[2] M. Akram, M. Iqbal, M. Daniyal, A.U. Khan, "Awareness and current knowledge of breast cancer". Biological Research vol, 50(1), pp. 1-23, 2017.

[3] A. Thorarinsson et al. "Long-term health-realted quality of life after breast reconstruction: Comparing 4 different methods of reconstruction". PRS Global Open, vol. 5(6) pp. 5-9, 2017.

[4] K.B. Santosa et al. "Long-term patient-reported outcomes in postmastectomy breast reconstruction". JAMA Surgery, Vol. 153(10), pp. 891-899, 2018.

[5] K. Sekiguchi, J. Kawamori, and H. Yamauchi. "Breast reconstruction and postmastectomy radiotherapy: complications by type and timing and other problems in radiation oncology". Breast Cancer, vol. 24(4), pp. 511-520, 2017.

[6] S.S. Kroll "Breast reconstruction with autologous tissue - Art and artistry".Springer-Verlag New York, ISBN: 978-0-387-98670-8, 2000.

[7] M. Barry and M.R. Kell. "Radiotherapy and breast reconstruction: A meta-analysis". Breast cancer research and treatment, vol. 127(1), pp. $15-22,2011$

[8] A.Y. Ho, Z.I Hu, B.J Mehrara, E.G. Wilkins, "Radiotherapy in the setting of breast reconstruction: types, techniques, and timing". The Lancet Oncology, vol. 18(12), pp. e742-e753, 2017.

[9] R. Jagsi et al. "Impact of radiotherapy on complications and patientreported outcomes after breast reconstruction". Journal of the National Cancer Institute, vol. 110(2), pp. 157-165, 2017.
[10] F.C. Wei and S. Mardini "Flaps and reconstruction surgery". American Journal of Sociology, Second vol. 53(9), pp. 1689-1699, 2019.

[11] A. Pawan "Breast reconstruction following mastectomy". Indian Journal of Surgery, vol. 66(4), pp. 249, 2004.

[12] S.K.F. Seal, M.L. Martin, P.M.A. Brasher, M.K. Hewitt, "Preoperative and postoperative assessment of Rectus Abdominis Muscle size and function following Deep Inferior Epigastric Perforator (DIEP) surgery". Plastic and Reconstructive Surgery 141(5), pp. 1, 2018

[13] M. Renzulli et al. "Preoperative Computed Tomography assessment for a Deep Inferior Epigastric Perforator (DIEP) flap: a new easy technique from the Bologna experience". Acta Radiologica, vol. 1, pp. 1-7, 2020.

[14] Clavero et al. "MDCT in the preoperative planning of abdominal perforator surgery for postmastectomy breast reconstruction". American Journal of Roentgenology 191, pp. 670-676, 2008.

[15] R.G. Wade et al. "Perforator mapping reduces the operative time of DIEP flap breast reconstruction: A systematic review and meta-analysis of preoperative Ultrasound, Computed Tomography and Magnetic Resonance Angiography". Journal of Plastic, Reconstructive and Aesthetic Surgery, vol. 71(4), pp. 468-477, 2018.

[16] H.F. Mayer "The use of a 3D simulator software and 3D printed biomodels to aid Autologous Breast Reconstruction". Aesthetic Plastic Surgery volume, vol. 44, pp. 1396-1402, 2020.

[17] X. Zhang et al. "Predicting the feasibility of utilizing SIEA flap for breast reconstruction with preoperative BMI and Computed Tomography Angiography (CTA) data” Aesth Plast Surg. vol. 45, pp. 100-107, 2021.

[18] G.D. Rosson et al. "Three-dimensional Computed Tomographic Angiography to predict weight and volume of Deep Inferior Epigastric Artery perforator flap for breast reconstruction" 56th annual meeting of the Plastic Surgery Research Council, Louisville, Kentucky, April 29, 2011.

[19] S. Mohan and Vasudevan Vani, "Multi-core computer vision and image processing for intelligent applications". IGI Global, pp. 1-291, 2016.

[20] S.L Bangare, A. Dubal, P.S. Bangare, S.T. Patil, "Reviewing Otsu's method for image thresholding". International Journal of Applied Engineering Research, 10(9), pp. 21777-21783, 2015.

[21] D. Liu and J. Yu "Otsu method and K-means". Ninth International Conference and Hybrid Intelligent Systems, 1, pp. 344-349, 2009.

[22] K.J. Woo, E.J. Kim, K.Tae. Lee, G.H. Mun, "A novel method to estimate the weight of the DIEP flap in breast reconstruction: DIEPW, a simple calculation formula using paraumbilical flap thickness". Journal of Reconstructive Microsurgery, vol. 32(7), pp. 520-527, 2016.

[23] J. Canny "A computational approach to edge detection". IEEE Transactions on Pattern Analysis and Machine Intelligence, 8(6), pp. 679-698, 1986.

[24] S. Rani, D. Bansal, B. Kaur, "Detection of edges using mathematical morphological operators". Open transactions on information processing, vol. 1(1), pp. 17-26, 2014.

[25] S. Razzano, R. Taylor, F. Schonauer, A. Figus, "How to assess the volume of a DIEP flap using a free online calculator: The DIEP $\mathrm{V}$ (volume) method". Journal of plastic, reconstruction and aesthetic surgery, vol. 71(10), pp. 1410-1416, 2018

[26] I. Matei et al. "Harvesting of forearm perforator flaps based on intraoperative vascular exploration: clinical experiences and literature review" Microsurgery, vol. 28(5), pp. 321-330, 2008

[27] A.B. Chagpar, "Prophylactic bilateral mastectomy and contralateral prophylactic mastectomy" Surgical Oncology Clinics, vol. 23(3), pp. 423-430, 2014.

[28] L. Drazan et al. "Bilateral breast reconstruction with DIEP flaps: 4 years' experience" Journal of Plastic Reconstructive and Aesthetic Surgery, vol. 61(11), pp. 1309-15, 2007

[29] W. He et al. "Complications and patient-reported outcomes after TRAM and DIEP flaps: A systematic review and meta-analysis". plast reconstruc surg Glob Open, vol. 8(10), pp. e3120, 2020.

[30] F. Timmermans et al. "A retrospective investigation of abdominal visceral fat, body mass index (BMI), and active smoking as risk factors for donor site wound healing complications after free DIEP flap breast reconstructions". Journal of Plastic Reconstructive and Aesthetic Surgery, vol. 71(6), pp. 1-6, 2018.

[31] T.K. Ghosh et al. "Multi-class probabilistic Atlas-Based whole heart segmentation method in cardiac CT and MRI". IEEE Access, vol. 9, pp. 66948-66964, 2021

[32] J. Wu and X. Tang. "Brain segmentation based on multi-atlas and diffeomorphism guided 3D fully convolutional network ensembles". Pattern Recognition, vol. 115, pp. 107904, 2021. 